\title{
The Epidemiology of Guillain-Barré Syndrome Worldwide
}

\author{
A Systematic Literature Review
}

\author{
Anita McGrogan ${ }^{a}$ Gemma C. Madle ${ }^{b}$ Helen E. Seaman ${ }^{b}$ Corinne S. de Vries ${ }^{a}$ \\ aDepartment of Pharmacy and Pharmacology, University of Bath, Bath, and \\ ${ }^{b}$ Department of Pharmacoepidemiology, Postgraduate Medical School, University of Surrey, Guildford, UK
}

\section{Key Words}

Epidemiology · Guillain-Barré syndrome $\cdot$ Incidence $\cdot$

Systematic review

\begin{abstract}
Background: This systematic literature review of the epidemiology of Guillain-Barré syndrome (GBS) identifies trends in incidence rates by age, study method and cause of disease. It is important to have a reliable estimate of incidence to determine and investigate any changes: no previous systematic reviews of GBS have been found. Methods: After critical assessment of the reliability of the reported data, incidence rates were extracted from all relevant papers published between 1980 and 2008, identified through searches of Medline, Embase and Science Direct. Results: Sixty-three papers were included in this review; these studies were prospective, retrospective reviews of medical records or retrospective database studies. Ten studies reported on the incidence in children (0-15 years old), and found the annual incidence to be between 0.34 and 1.34/100,000. Most studies investigated populations in Europe and North America and reported similar annual incidence rates, i.e. between 0.84 and 1.91/100,000. A decrease in incidence over the time between the 1980s and 1990s was found. Up to $70 \%$ of cases of GBS were caused by antecedent infections. Conclusions: Our best estimate of the overall incidence of GBS
\end{abstract}

was between 1.1/100,000/year and 1.8/100,000/year. The incidence of GBS increased with age after 50 years from $1.7 / 100,000 /$ year to $3.3 / 100,000 /$ year.

Copyright $\odot 2008$ S. Karger AG, Basel

\section{Introduction}

Guillain-Barré syndrome (GBS) is a peripheral neuropathy with acute onset, and characterised by rapidly developing motor weakness [1-3]. The disease is thought to be autoimmune and triggered by a preceding infection in two thirds of cases, most frequently respiratory or gastrointestinal infections $[3,4]$. Possible links between vaccinations and the occurrence of cases of GBS have been proposed, although the evidence for this link is not strong. An increase in GBS incidence of about 1 case per million above background incidence has been associated with the 1976 New Jersey swine influenza vaccination programme, and of about 1 case per thousand associated with rabies vaccinations $[3,5]$.

GBS can be divided into at least 4 main subtypes of disease: acute inflammatory demyelinating polyradiculoneuropathy (AIDP), the axonal subtypes, i.e. acute motor axonal neuropathy (AMAN) and acute motor and sensory axonal neuropathy (AMSAN), and Miller Fisher syndrome, the main symptoms of which are oculomotor

\section{KARGER \\ Fax +4161306 1234 E-Mail karger@karger.ch} www.karger.com (c) 2008 S. Karger AG, Basel 0251-5350/09/0322-0150\$26.00/0

Accessible online at:

www.karger.com/ned
Anita McGrogan

Department of Pharmacy and Pharmacology

University of Bath

Bath BA2 7AY (UK)

Tel. +44 1225384 228, Fax +44 1225386 114, E-Mail a.mcgrogan@bath.ac.uk 
dysfunction, ataxia and areflexia. In North America and Europe, around $5 \%$ of patients with GBS have the axonal subtypes, whereas in Central and South America, Japan and China axonal subtypes account for $30-47 \%$ of cases [5]; Miller-Fisher syndrome has been found to account for around $5 \%$ of cases of GBS $[3,5]$. Standard criteria for the diagnosis of GBS were published in 1978 by a National Institute of Neurological and Communicative Diseases (NINCDS) committee. The criteria include clinical features such as progression, relative symmetry and mild sensory symptoms or signs as well as levels of protein in cerebrospinal fluid, and electrodiagnostic features, such as nerve conduction slowing or blocking [2]. Typical treatment regimens for GBS include plasma exchange and intravenous immunoglobulin. Both treatments are thought to be equally efficacious, but Tsai et al. [6] showed that intravenous immunoglobulin may be cheaper due to patients having fewer complications and requiring a shorter stay in hospital; direct health care costs were estimated to be approximately USD 110,000 in the USA.

Insight into the incidence of disease is important for the identification of trends in relation to patient characteristics, such as age and geographical location, and to determine any changes in incidence following exposure to new environmental factors. Previous reviews evaluating the incidence of GBS have found rates to be between 0.16 and 4.0/100,000/year in individuals of all ages $[3,4]$ and between 0.5 and 1.5/100,000/year in those under 18 years [7]. The highest rates have been reported in adults, especially those aged over 75 years $[4,5]$. Unusually for an autoimmune disease, higher incidence rates have been reported in males than females [4].

To our knowledge, no systematic evaluation of published studies of the incidence of GBS has been published. Previous epidemiological reviews of GBS include: Sladky [7] who reported on incidence of GBS in children, Govoni and Granieri [3] who included studies published between 1978 and 2000 and described overall trends in incidence rates, and Hughes and Rees [4] who tabulated rates from 35 studies published between 1958 and 1996. The objectives of this literature review were to identify reliable estimates of GBS incidence, to compare rates by age and geography, and to examine any changes in incidence over time.

\section{Method}

Searches of the Medline (1980-2008), EMBASE (1980-2008) and Science Direct (1980-2008) databases were carried out using the keywords ‘Guillain-Barré Syndrome' or 'polyradiculoneurop- athy' and 'incidence' or 'epidemiology'. The 2 disease terms were used because the MeSH term 'Guillain-Barré syndrome' was only used in Medline from 1998.

The inclusion criteria were that the studies reported original work, that a reasonable effort had been made by the authors to include all incident cases and that the estimates of population size and person-time contributed during the study period were accurate. When assessing the likelihood of missing incident cases, papers were evaluated as follows: (1) for case-finding studies, did the authors ensure that all of the subjects contributing person-time to the denominator data were available as potential cases and did the authors check all relevant medical records? (2) for all studies, were cases checked to ensure that they were incident and not prevalent? (3) for all studies, did the authors ensure that the cause of GBS was autoimmune and it was not secondary to another disease?

The titles and abstracts (where available) of all of the studies identified by the searches were reviewed by 2 investigators. Studies published in English, French, German, Spanish or Dutch were included. The full text of all potentially relevant papers was appraised and decisions about inclusion of papers were discussed by all authors. Review papers were searched for secondary references reporting on original research; secondary references found from any of the other papers reviewed were also included.

A standard data abstraction form was used to record all details of the papers reviewed (appendix 1). Each study was classified as being at low, medium or high risk of under- or overestimation of reported incidence rates by considering the reliability of numerator and denominator data. For instance, inclusion of prevalent cases or those thought not to be caused by autoimmunity will have led to overestimated rates, as will underestimated denominator data. Conversely, missing cases or an overestimated denominator (e.g. a catchment area from which not all inhabitants had access to hospital services) would be considered to result in underestimated incidence rates. Explanations provided by the papers' authors as to why incidence rates were as expected or whether they were considered to be an over- or underestimate of the true incidence rate were taken into account in this process. If the extent of likely error was considered to be very great, the study was excluded. To minimise subjectivity, this assessment was agreed between the authors and random checks were performed to ensure consistency. Rates are presented as the number of cases/100,000/year and where sufficient data were given in the paper, rates were checked for accuracy.

\section{Results}

Figure 1 shows the results of the database searches and subsequent filtering of search results. A list of all of the references considered for this study but excluded is available on request from the authors.

Of the 457 papers rejected at the abstract review stage, nearly half did not focus on GBS as the subject of the paper and one fifth did not investigate incidence of GBS; other key reasons for rejecting papers at this stage included those reviewing the disease, case series, those investigating outcomes of vaccinations and those reporting on incidence of a disease other than GBS. Following review 
Fig. 1. Results from Medline search.

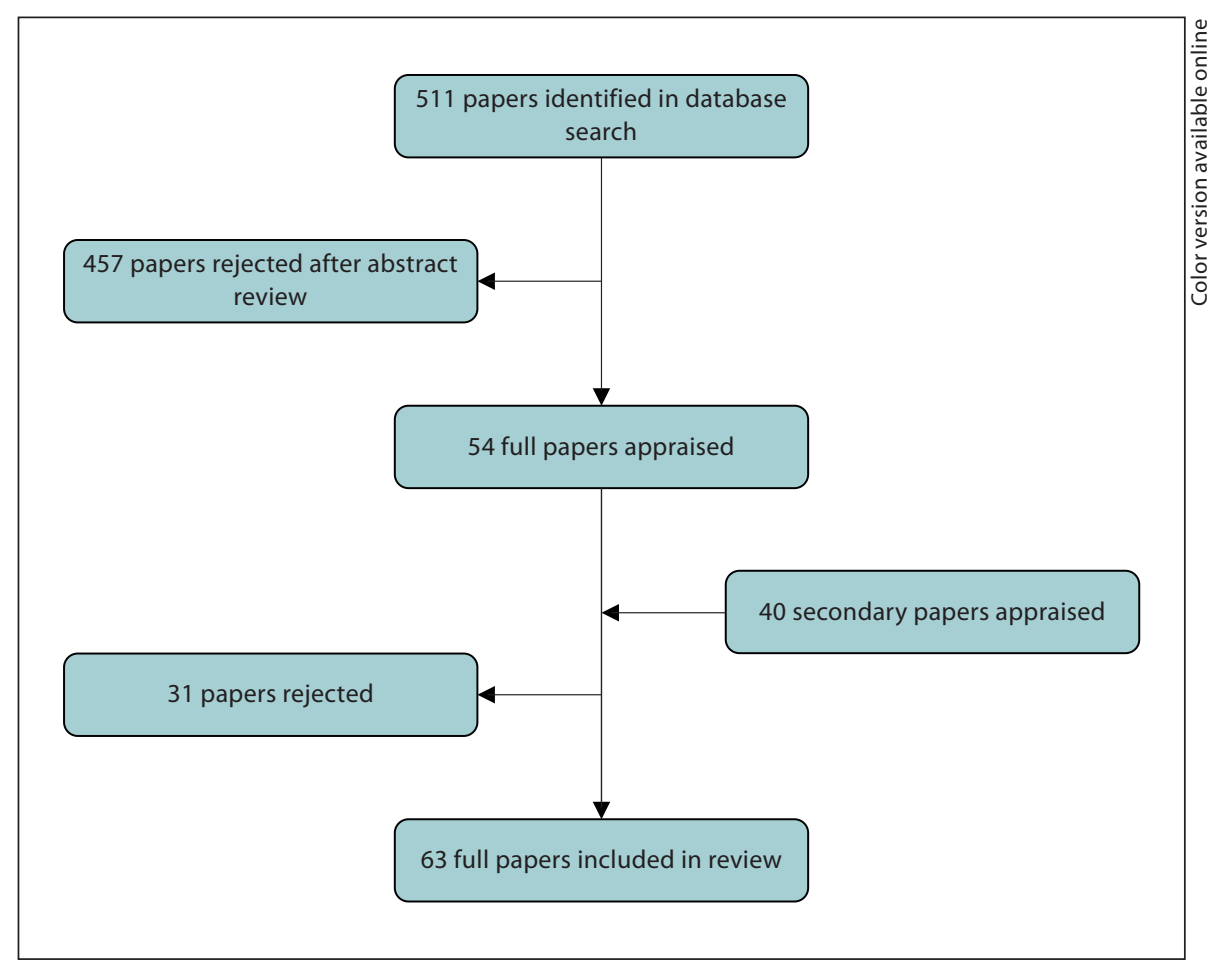

of the full papers, 31 papers were rejected; key reasons for this included papers that reviewed the disease and did not provide primary data on incidence, papers reporting data given in other papers already included, incidence rates not presented and too few data given to calculate incidence rates and papers reporting incidence data thought to be very unreliable. Each of the 63 studies included in this review is described in table 1 , first for children and then for adults grouped by continent. Incidence rates broken down by age band are given in table 2 .

\section{Incidence Rates}

The incidence rates found for GBS varied between $0.38 / 100,000 /$ year (95\% CI 0.25-0.56) in Finland [8] and 2.53/100,000/year (95\% CI 1.87-3.35) in Curaçao [9] (table 1).

\section{Geographical Variation}

Most of the studies included in this review were from Europe and North America where the majority of incidence rates were between 0.84/100,000/year (Finland) [10] and 1.91/100,000/year (Italy) [11]. For other parts of the world, very few studies were found, and therefore it is difficult to comment on any geographical trends. For the data presented, a comparison of these rates with those of Europe and North America suggest that incidence was lower in China [12, 13], Hong Kong [14] and Brazil [15], similar in Tanzania [21], Australia [16, 17] and Japan [18] and slightly higher in the Middle East [19, 20] and Curaçao [9].

\section{Variation over Time}

Most studies covered time periods between 1980 and 2000 [9-19, 21-43]. Between 1980 and 2000, the incidence was between 1.0/100,000/year and 1.8/100,000/ year. Only 3 studies reported rates from 2000 onwards, and these were thought to be unreliable $[20,44,45]$.

\section{Variation with Age}

In the majority of studies that included incidence rates broken down by age, increases in rates were observed in most studies of people aged 50 years or more [11, 17, 22, $26,31-33,35-39,46-49]$, with some showing a decrease in the highest age group of $\geq 80$ years $[22,26,31,35-38$, 47-49].

Overall incidence rates in children ranged from $0.34 / 100,000 /$ year to $1.34 / 100,000 /$ year $[31,34]$. In the studies that investigated incidence in children (0-9 years) and teenagers (10-19 years), most showed an increase in incidence with increasing age $[19,37,38,48-51]$, although some demonstrated decreases $[12,13,17,46,47]$. 
Table 1. Description of studies and overall incidence by continent

\begin{tabular}{|c|c|c|c|c|c|c|c|c|}
\hline Study & Location & Study method & $\begin{array}{l}\text { Diagnostic } \\
\text { criteria }\end{array}$ & $\begin{array}{l}\text { Cases } \\
\mathrm{n}\end{array}$ & $\begin{array}{l}\text { Antecedent } \\
\text { infection, \% }\end{array}$ & Period & Age & $\begin{array}{l}\text { Rate } \\
100,000 / \text { year }\end{array}$ \\
\hline \multicolumn{9}{|l|}{ Children } \\
\hline $\begin{array}{l}\text { Hung et al. [60] } \\
\text { RUE: }{ }^{*} \\
\text { ROE: }{ }^{*}\end{array}$ & Taiwan & $\begin{array}{l}\text { medical record review by paediatric } \\
\text { neurologists }\end{array}$ & NINCDS & 72 & $\begin{array}{l}\text { all: } 83 \\
\text { URI: } 68 \\
\text { GI: } 7 \\
\text { other: } 7\end{array}$ & $1986-90$ & $0-16$ & 0.66 \\
\hline $\begin{array}{l}\text { Rantala et al. [8] } \\
\text { RUE: * } \\
\text { ROE: * }\end{array}$ & Finland & $\begin{array}{l}\text { hospital discharge database; diagnoses } \\
\text { of cases reviewed }\end{array}$ & not given & 27 & $\begin{array}{l}\text { all: } 85 \\
\text { URI: } 67\end{array}$ & $1980-86$ & $\leq 15$ & $\begin{array}{l}0.38 \\
(0.25-0.56)^{1}\end{array}$ \\
\hline $\begin{array}{l}\text { Artan }[61] \\
\text { RUE: * } \\
\text { ROE: * }\end{array}$ & $\begin{array}{l}\text { Antalya, } \\
\text { Turkey }\end{array}$ & $\begin{array}{l}\text { retrospective study of cases of acute } \\
\text { paralysis reported to Ministry of Health }\end{array}$ & $\begin{array}{l}\text { Asbury and } \\
\text { Cornblath }\end{array}$ & 11 & URI: 63 & $1990-96$ & $1-14$ & 0.54 \\
\hline $\begin{array}{l}\text { Barzegar et al. [53] } \\
\text { RUE: ** } \\
\text { ROE: * }\end{array}$ & $\begin{array}{l}\text { Eastern } \\
\text { Azerbaijan, } \\
\text { Iran }\end{array}$ & $\begin{array}{l}\text { systematic registration of children with } \\
\text { acute flaccid paralysis; all cases referred. }\end{array}$ & $\begin{array}{l}\text { Asbury and } \\
\text { Cornblath }\end{array}$ & 143 & $\begin{array}{l}\text { all: } 69 \\
\text { URI: } 52 \\
\text { GI: } 14 \\
\text { other: } 3\end{array}$ & 2001-06 & $0-15$ & 2.27 \\
\hline $\begin{array}{l}\text { Ismail et al. [67] } \\
\text { RUE: ** } \\
\text { ROE: * }\end{array}$ & Kuwait & $\begin{array}{l}\text { retrospective review of medical records } \\
\text { identifying hospital discharges coded } \\
\text { for GBS; records reviewed }\end{array}$ & $\begin{array}{l}\text { Asbury and } \\
\text { Cornblath }\end{array}$ & 19 & $\begin{array}{l}\text { all: } 79 \\
\text { URI: } 68 \\
\text { GI: } 11\end{array}$ & 1992-97 & $\leq 12$ & $\begin{array}{l}0.95 \\
(0.52-1.37)^{1,2}\end{array}$ \\
\hline $\begin{array}{l}\text { Molinero et al. [57] } \\
\text { RUE: }{ }^{*} \\
\text { ROE: } * * *\end{array}$ & Honduras & prospective hospital-based study & $\begin{array}{l}\text { Asbury and } \\
\text { Cornblath }\end{array}$ & 394 & not given & 1989-99 & $<16$ & $\begin{array}{l}1.37 \\
(1.22-1.49)^{1,2}\end{array}$ \\
\hline $\begin{array}{l}\text { Rantala et al. [68] } \\
\text { RUE: } \\
\text { ROE: }\end{array}$ & $\begin{array}{l}\text { Los Angeles } \\
\text { and Orange } \\
\text { Counties, } \\
\text { USA }\end{array}$ & $\begin{array}{l}\text { medical records of those discharged } \\
\text { from children's hospitals; case histories } \\
\text { reviewed by paediatric neurologist }\end{array}$ & $\begin{array}{l}\text { review of cases } \\
\text { with ICD } \\
356.0-357.9\end{array}$ & 93 & $\begin{array}{l}\text { all: } 76 \\
\text { URI: } 48 \\
\text { GI: } 12 \\
\text { other: } 11\end{array}$ & $1980-86$ & $\leq 15$ & $\begin{array}{l}0.60 \\
(0.48-0.73)^{1}\end{array}$ \\
\hline $\begin{array}{l}\text { Olive et al. [58] } \\
\text { RUE: }{ }^{*} \\
\text { ROE: }{ }^{*}\end{array}$ & $\begin{array}{l}\text { South } \\
\text { America }\end{array}$ & $\begin{array}{l}\text { all cases of acute flaccid paralysis must } \\
\text { be reported for surveillance; active } \\
\text { searches: door to door survey, hospital } \\
\text { admissions, death certificates; more } \\
\text { detailed studies in } 7 \text { countries }\end{array}$ & NINCDS & 3,112 & all: 67 & $1989-91$ & $<15$ & 0.67 \\
\hline $\begin{array}{l}\text { Dias-Tosta et al. [54] } \\
\text { RUE: * } \\
\text { ROE: ** }\end{array}$ & Brazil & $\begin{array}{l}\text { cases identified from the Fundação } \\
\text { Nacional de Saúde of the Brazilian Min- } \\
\text { istry of Health; data collected during a } \\
\text { poliomyelitis surveillance programme } \\
\text { reviewed and 60-day follow-up }\end{array}$ & $\begin{array}{l}\text { Asbury and } \\
\text { Cornblath }\end{array}$ & 1,678 & not given & $1990-96$ & $<15$ & 0.46 \\
\hline $\begin{array}{l}\text { Hart et al. [59] } \\
\text { RUE: * } \\
\text { ROE: * }\end{array}$ & Paraguay & $\begin{array}{l}\text { compulsory reporting of GBS cases to } \\
\text { the Ministry of Health; cases also identi- } \\
\text { fied through door-to-door vaccination } \\
\text { programs and a notification agreement } \\
\text { with neighbouring countries; records } \\
\text { reviewed }\end{array}$ & NINCDS & 37 & $\begin{array}{l}\text { all: } 54 \\
\text { URI: } 32 \\
\text { GI: } 8 \\
\text { other: } 14\end{array}$ & $1990-91$ & $<15$ & $\begin{array}{l}1.06 \\
(0.72-1.40)^{1,2}\end{array}$ \\
\hline \multicolumn{9}{|l|}{ All ages } \\
\hline $\begin{array}{l}\text { Africa } \\
\text { Howlett et al. [21] } \\
\text { RUE: ** } \\
\text { ROE: * }\end{array}$ & $\begin{array}{l}\text { Kilimanjaro, } \\
\text { Tanzania }\end{array}$ & $\begin{array}{l}\text { retrospective hospital-based study with } \\
\text { review of medical records (ICD 357.0) }\end{array}$ & NINCDS & 45 & all: 40.6 & 1984-92 & $\geq 12$ & 0.83 \\
\hline \multicolumn{9}{|l|}{ Asia } \\
\hline $\begin{array}{l}\text { Cheng et al. [12] } \\
\text { RUE: * } \\
\text { ROE: * }\end{array}$ & $\begin{array}{l}\text { Harbin, } \\
\text { China }\end{array}$ & $\begin{array}{l}\text { prospective reporting of new cases } \\
\text { by hospitals and clinics; cases and } \\
\text { admission registers checked }\end{array}$ & NINCDS & 36 & $\begin{array}{l}\text { all: } 75 \\
\text { URI: } 58 \\
\text { GI: } 11 \\
\text { other: } 6\end{array}$ & 1997-98 & all & $\begin{array}{l}0.66 \\
(0.46-0.91)^{3}\end{array}$ \\
\hline $\begin{array}{l}\text { Zhang et al. [13] } \\
\text { RUE: * } \\
\text { ROE: * }\end{array}$ & $\begin{array}{l}\text { Harbin, } \\
\text { China }\end{array}$ & $\begin{array}{l}\text { surveillance system: new cases were } \\
\text { reported to the study; checks for } \\
\text { missing cases also conducted }\end{array}$ & $\begin{array}{l}\text { Asbury and } \\
\text { Cornblath }\end{array}$ & 72 & $\begin{array}{l}\text { all: } 71 \\
\text { URI: } 58 \\
\text { GI: } 4 \\
\text { URI and GI: } 9\end{array}$ & 1997-99 & all & $0.69^{3}$ \\
\hline $\begin{array}{l}\text { Hui et al. [14] } \\
\text { RUE: }{ }^{* *} \\
\text { ROE: }{ }^{* *}\end{array}$ & $\begin{array}{l}\text { Hong Kong, } \\
\text { China }\end{array}$ & $\begin{array}{l}\text { retrospective review of admission and } \\
\text { discharge records; clinical variants } \\
\text { included }\end{array}$ & $\begin{array}{l}\text { ICD-9 codes } \\
357.0,356.4 \\
356.8,356.9\end{array}$ & 20 & GI: 25 & 1993-98 & $>15$ & $\begin{array}{l}0.44 \\
(0.25-0.64)^{1,2}\end{array}$ \\
\hline $\begin{array}{l}\text { Kusumi et al. [18] } \\
\text { RUE: }{ }^{*} \\
\text { ROE: }{ }^{*}\end{array}$ & $\begin{array}{l}\text { Tottori } \\
\text { Prefecture, } \\
\text { Japan }\end{array}$ & $\begin{array}{l}\text { patient records at the university } \\
\text { hospital; questionnaires sent to } \\
\text { general hospitals }\end{array}$ & NINCDS & 35 & not given & 1988-92 & all & 1.14 \\
\hline
\end{tabular}


Table 1 (continued)

\begin{tabular}{|c|c|c|c|c|c|c|c|c|}
\hline Study & Location & Study method & $\begin{array}{l}\text { Diagnostic } \\
\text { criteria }\end{array}$ & $\begin{array}{l}\text { Cases } \\
\mathrm{n}\end{array}$ & $\begin{array}{l}\text { Antecedent } \\
\text { infection, } \%\end{array}$ & Period & Age & $\begin{array}{l}\text { Rate } \\
100,000 / \text { year }\end{array}$ \\
\hline \multicolumn{9}{|l|}{ Australasia } \\
\hline $\begin{array}{l}\text { Storey et al. [16] } \\
\text { RUE: ** } \\
\text { ROE: * }\end{array}$ & $\begin{array}{l}\text { Victoria, } \\
\text { Australia }\end{array}$ & $\begin{array}{l}\text { medical record systems in teaching } \\
\text { hospitals; all cases reviewed }\end{array}$ & $\begin{array}{l}\text { criteria not } \\
\text { given }\end{array}$ & 110 & $\begin{array}{l}\text { URI: } 34 \\
\text { GI: } 12\end{array}$ & $1980-84$ & $\geq 15$ & $0.90^{3}$ \\
\hline $\begin{array}{l}\text { Hankey [17] } \\
\text { RUE: }{ }^{* *} \\
\text { ROE: }{ }^{*}\end{array}$ & $\begin{array}{l}\text { Western } \\
\text { Australia }\end{array}$ & $\begin{array}{l}\text { case record search for GBS, polyneuritis } \\
\text { or polyneuropathy codes }\end{array}$ & Asbury & 109 & all: 58 & $1980-85$ & all & $\begin{array}{l}1.35 \\
(1.10-1.61)^{1,2}\end{array}$ \\
\hline \multicolumn{9}{|l|}{ The Caribbean } \\
\hline $\begin{array}{l}\text { van Koningsveld et al. [9] } \\
\text { RUE: * } \\
\text { ROE: }{ }^{*}\end{array}$ & Curaçao & $\begin{array}{l}\text { medical records with relevant ICD-9 } \\
\text { codes; cases reviewed }\end{array}$ & NINCDS & 49 & $\begin{array}{l}\text { all: } 92 \\
\text { URI: } 5 \\
\text { influenza: } 25 \\
\text { GI: } 55 \\
\text { other: } 7\end{array}$ & $1987-96$ & all & $\begin{array}{l}2.53 \\
(1.87-3.35)^{1}\end{array}$ \\
\hline \multicolumn{9}{|l|}{ Europe } \\
\hline $\begin{array}{l}\text { Bak [62] } \\
\text { RUE: }{ }^{*} \\
\text { ROE: }{ }^{* *}\end{array}$ & $\begin{array}{l}\text { Ringkobing } \\
\text { County, } \\
\text { Denmark }\end{array}$ & $\begin{array}{l}\text { record review for patients admitted to } \\
\text { all hospitals in the county. }\end{array}$ & $\begin{array}{l}\text { Danish ICD } \\
\text { code for GBS } \\
\text { (ICD adaptation) }\end{array}$ & 51 & all: 41 & $1965-82$ & all & $1.14^{3}$ \\
\hline $\begin{array}{l}\text { Halls et al. [63] } \\
\text { RUE: }{ }^{* *} \\
\text { ROE: }{ }^{* *}\end{array}$ & $\begin{array}{l}\text { Copenhagen } \\
\text { county, } \\
\text { Denmark }\end{array}$ & $\begin{array}{l}\text { record review of those with a diagnosis } \\
\text { of neuropathy, to find cases of GBS }\end{array}$ & $\begin{array}{l}\text { criteria not } \\
\text { given }\end{array}$ & 34 & not given & $1977-84$ & all & $\begin{array}{l}1.50 \\
(0.90-2.30)^{3}\end{array}$ \\
\hline $\begin{array}{l}\text { Hughes et al. [22] } \\
\text { RUE: * } \\
\text { ROE: }{ }^{*}\end{array}$ & UK & $\begin{array}{l}\text { population-based study using General } \\
\text { Practice Research database }\end{array}$ & $\begin{array}{l}\text { codes for GBS } \\
\text { or infective } \\
\text { neuritis used }\end{array}$ & 228 & not given & 1992-2000 & all & $\begin{array}{l}1.33 \\
(1.15-1.50)^{1,3}\end{array}$ \\
\hline $\begin{array}{l}\text { MacDonald et al. [23] } \\
\text { RUE: }{ }^{*} \\
\text { ROE: }{ }^{*}\end{array}$ & $\begin{array}{l}\text { London, } \\
\text { England }\end{array}$ & $\begin{array}{l}\text { case ascertainment using referrals, } \\
\text { databases, clinic records and patient } \\
\text { medical records }\end{array}$ & $\begin{array}{l}\text { diagnostic } \\
\text { criteria not } \\
\text { given }\end{array}$ & $\begin{array}{l}\text { not } \\
\text { given }\end{array}$ & not given & 1995-96 & all & $\begin{array}{l}3.0 \\
(1.0-6.0)^{1,4}\end{array}$ \\
\hline $\begin{array}{l}\text { Haberman et al. [64] } \\
\text { RUE: ** } \\
\text { ROE: * }\end{array}$ & $\begin{array}{l}\text { North-West } \\
\text { Thames, } \\
\text { England }\end{array}$ & $\begin{array}{l}\text { retrospective review of hospital activity } \\
\text { analysis }\end{array}$ & $\begin{array}{l}\text { ICD- } 8 \text { code } \\
354.9 \text {, clinical fea- } \\
\text { tures, diagnosed } \\
\text { by a neurologist }\end{array}$ & 39 & not given & 1978 & all & $\begin{array}{l}1.10 \\
(0.77-1.48)^{1}\end{array}$ \\
\hline $\begin{array}{l}\text { Winner et al. [46] } \\
\text { RUE: * } \\
\text { ROE: * }\end{array}$ & $\begin{array}{l}\text { Oxfordshire, } \\
\text { England }\end{array}$ & $\begin{array}{l}\text { hospital records and those from record } \\
\text { linkage study used with discharge codes } \\
\text { for GBS or acute infective polyneuritis } \\
\text { (ICD-8 354, ICD-9 357.0, 357.9) }\end{array}$ & $\begin{array}{l}\text { NINCDS, MF } \\
\text { also included } \\
\text { (Asbury) }\end{array}$ & 72 & not given & 1978 & all & $\begin{array}{l}1.10 \\
(0.80-1.40)^{1}\end{array}$ \\
\hline $\begin{array}{l}\text { Rees et al. [24] } \\
\text { RUE: }{ }^{*} \\
\text { ROE: }{ }^{*}\end{array}$ & $\begin{array}{l}\text { south-east } \\
\text { England }\end{array}$ & $\begin{array}{l}\text { prospective reporting of cases, hospital } \\
\text { activity analysis, research database and } \\
\text { death certificates }\end{array}$ & $\begin{array}{l}\text { Asbury and } \\
\text { Cornblath } \\
\text { criteria used }\end{array}$ & 79 & not given & 1993-94 & all & $\begin{array}{l}1.20 \\
(0.90-1.40)^{1}\end{array}$ \\
\hline $\begin{array}{l}\text { Kinnunen et al. [10] } \\
\text { RUE: }{ }^{*} \\
\text { ROE: }{ }^{*}\end{array}$ & Finland & $\begin{array}{l}\text { cases with diagnosis of polyradiculitis } \\
\text { found from hospital discharge database } \\
\text { and reviewed by a neurologist }\end{array}$ & $\begin{array}{l}\text { Asbury and } \\
\text { Poser criteria } \\
\text { used }\end{array}$ & 247 & all: 67 & $1981-86$ & all & $\begin{array}{l}0.84 \\
(0.56-1.25)^{1}\end{array}$ \\
\hline $\begin{array}{l}\text { Lehmann [44] } \\
\text { RUE: }{ }^{* *} \\
\text { ROE: }{ }^{* * *}\end{array}$ & Germany & $\begin{array}{l}\text { nationwide administrative database } \\
\text { from reimbursement scheme imple- } \\
\text { mentation }\end{array}$ & $\begin{array}{l}\text { ICD-10 code } \\
\text { G61.0 used to } \\
\text { find cases }\end{array}$ & 4,349 & not given & $\begin{array}{l}2003 \\
2004 \\
2005\end{array}$ & all & $\begin{array}{l}1.78^{2} \\
1.6^{2} \\
1.89^{2}\end{array}$ \\
\hline $\begin{array}{l}\text { Markoula et al. [25] } \\
\text { RUE: ** } \\
\text { ROE: * }\end{array}$ & $\begin{array}{l}\text { north-west } \\
\text { Greece }\end{array}$ & $\begin{array}{l}\text { records of patients admitted to neurol- } \\
\text { ogy inpatients reviewed; variants were } \\
\text { included }\end{array}$ & NINCDS & 46 & $\begin{array}{l}\text { all: } 61 \\
\text { URI: } 30 \\
\text { GI: } 26\end{array}$ & 1996-2005 & all & 1.22 \\
\hline $\begin{array}{l}\text { Chroni et al. [26] } \\
\text { RUE: }{ }^{*} \\
\text { ROE: }{ }^{*}\end{array}$ & $\begin{array}{l}\text { south-west } \\
\text { Greece }\end{array}$ & $\begin{array}{l}\text { retrospective review of medical records; } \\
\text { many cases followed up after } 14 \\
\text { months. }\end{array}$ & NINCDS & 105 & $\begin{array}{l}\text { all: } 50 \\
\text { URI: } 29 \\
\text { GI: } 7 \\
\text { other: } 14\end{array}$ & 1989-2001 & all & $\begin{array}{l}0.99 \\
(0.81-1.19)^{1}\end{array}$ \\
\hline $\begin{array}{l}\text { Anon }[27,47] \\
\text { RUE: }{ }^{*} \\
\text { ROE: }\end{array}$ & $\begin{array}{l}\text { Emilia- } \\
\text { Romagna, } \\
\text { Italy }\end{array}$ & $\begin{array}{l}\text { prospective study of all neurological } \\
\text { units and relevant departments; } \\
\text { discharges using ICD codes were also } \\
\text { checked }\end{array}$ & not given & 105 & $\begin{array}{l}\text { all: } 70 \\
\text { URI: } 46 \\
\text { influenza: } 13 \\
\text { GI: } 11\end{array}$ & $\begin{array}{l}\text { 1992-93 } \\
\text { NINCDS } \\
\text { GBS and } \\
\text { variants }\end{array}$ & all & $\begin{array}{l}1.11 \\
(0.89-1.36)^{1} \\
1.2 \\
(0.961 .46)^{1,3}\end{array}$ \\
\hline $\begin{array}{l}\text { Paolino et al. [28] } \\
\text { RUE: * } \\
\text { ROE: * }\end{array}$ & $\begin{array}{l}\text { Ferrara, } \\
\text { Italy }\end{array}$ & $\begin{array}{l}\text { medical records from relevant depart- } \\
\text { ments searched; diagnosis of GBS and } \\
\text { related disorders checked }\end{array}$ & NINCDS & 16 & $\begin{array}{l}\text { all: } 38 \\
\text { URI: } 19 \\
\text { GI: } 6 \\
\text { other: } 13\end{array}$ & $1981-87$ & all & $\begin{array}{l}1.26 \\
(0.65-1.88)^{1,2}\end{array}$ \\
\hline
\end{tabular}


Table 1 (continued)

\begin{tabular}{|c|c|c|c|c|c|c|c|c|}
\hline Study & Location & Study method & $\begin{array}{l}\text { Diagnostic } \\
\text { criteria }\end{array}$ & $\begin{array}{l}\text { Cases } \\
\mathrm{n}\end{array}$ & $\begin{array}{l}\text { Antecedent } \\
\text { infection, } \%\end{array}$ & Period & Age & $\begin{array}{l}\text { Rate } \\
100,000 / \text { year }\end{array}$ \\
\hline $\begin{array}{l}\text { Govoni et al. }[11,29] \\
\text { RUE: * } \\
\text { ROE: * }\end{array}$ & $\begin{array}{l}\text { Ferrara, } \\
\text { Italy }\end{array}$ & $\begin{array}{l}\text { prospective identification of patients } \\
\text { referred to neurological wards; retro- } \\
\text { spective review of medical records and } \\
\text { discharge records (ICD-9 356.9, 357.0, } \\
356.4,356.8 \text { ) }\end{array}$ & $\begin{array}{l}\text { NINCDS (GBS); } \\
\text { Asbury and } \\
\text { Cornblath } \\
\text { (variants) }\end{array}$ & $\begin{array}{l}43[29], \\
26[11]\end{array}$ & $\begin{array}{l}\text { all: } 54 \\
\text { URI: } 23 \\
\text { GI: } 9 \\
\text { other: } 22\end{array}$ & $\begin{array}{l}1981-93 \\
{[29], 1994-} \\
2001[11]\end{array}$ & all & $\begin{array}{l}1.66^{3} \\
1.91 \\
(1.49-2.43)\end{array}$ \\
\hline $\begin{array}{l}\text { Beghi et al. [30] } \\
\text { RUE: * } \\
\text { ROE: * }\end{array}$ & $\begin{array}{l}\text { Lombardy, } \\
\text { Italy }\end{array}$ & $\begin{array}{l}\text { prospective hospital-based survey; } \\
\text { patients were interviewed and atypical } \\
\text { cases discussed }\end{array}$ & $\begin{array}{l}\text { NINCDS (GBS); } \\
\text { Ropper (variants) }\end{array}$ & 109 & not given & 1994-95 & all & $\begin{array}{l}0.92 \\
(0.75-1.09)^{1}\end{array}$ \\
\hline $\begin{array}{l}\text { Bogliun et al. [31] } \\
\text { RUE: ** } \\
\text { ROE: * }\end{array}$ & $\begin{array}{l}\text { Lombardy, } \\
\text { Italy }\end{array}$ & $\begin{array}{l}\text { cases traced through hospital, prospec- } \\
\text { tive regional registry started in } 1994 \text {; } \\
\text { hospital discharges also checked for } \\
\text { ICD-9 code } 357.0\end{array}$ & $\begin{array}{l}\text { NINCDS (GBS); } \\
\text { Asbury and Rop- } \\
\text { per (variants) }\end{array}$ & 138 & $\begin{array}{l}\text { all: } 30 \\
\text { URI: } 13 \\
\text { GI: } 7 \\
\text { influenza: } 10\end{array}$ & $1993-96$ & all & $\begin{array}{l}1.43 \\
(1.16-1.74)^{1}\end{array}$ \\
\hline $\begin{array}{l}\text { D’Ambrosio et al. [52] } \\
\text { RUE: *** } \\
\text { ROE: }{ }^{*}\end{array}$ & $\begin{array}{l}\text { Naples and } \\
\text { province, } \\
\text { Italy }\end{array}$ & $\begin{array}{l}\text { admission diagnoses reviewed and } \\
\text { records examined }\end{array}$ & NINCDS & 46 & not given & $1971-80$ & all & $\begin{array}{l}0.16 \\
(0.11-0.21)^{1,2}\end{array}$ \\
\hline $\begin{array}{l}\text { Chio et al. [32] } \\
\text { RUE: * } \\
\text { ROE: * }\end{array}$ & $\begin{array}{l}\text { Piedmont and } \\
\text { Valle d'Aosta, } \\
\text { Italy }\end{array}$ & $\begin{array}{l}\text { prospective recording in registry of } \\
\text { cases found in neurology departments; } \\
\text { hospital discharge databases also } \\
\text { checked for ICD-9 codes } 357.0,357.8 \\
\text { and } 357.9 \text {; cases verified }\end{array}$ & NINCDS & 120 & $\begin{array}{l}\text { all: } 58 \\
\text { URI/influenza: } \\
41 \\
\text { GI: } 14 \\
\text { other: } 4\end{array}$ & $1995-96$ & all & $\begin{array}{l}1.28 \\
(1.04-1.51)^{5}\end{array}$ \\
\hline $\begin{array}{l}\text { Congia et al. [55] } \\
\text { RUE: }{ }^{* * *} \\
\text { ROE: }{ }^{*}\end{array}$ & $\begin{array}{l}\text { Sardinia, } \\
\text { Italy }\end{array}$ & $\begin{array}{l}\text { retrospective review of medical records } \\
\text { and hospitalisations }\end{array}$ & NINCDS & 120 & all: 36 & $1961-80$ & all & $\begin{array}{l}0.40 \\
(0.33-0.47)^{1,2}\end{array}$ \\
\hline $\begin{array}{l}\text { van Koningsveld } \\
\text { et al. [33] } \\
\text { RUE: * } \\
\text { ROE: * }\end{array}$ & $\begin{array}{l}\text { south-west } \\
\text { Netherlands }\end{array}$ & $\begin{array}{l}\text { retrospective review of medical records } \\
\text { checking for ICD- } 9 \text { codes } 357.0,357.8 \\
\text { and } 357.9 \text {; cases re-evaluated by neurol- } \\
\text { ogist }\end{array}$ & NINCDS & 476 & $\begin{array}{l}\text { all: } 79 \\
\text { URI: } 22 \\
\text { GI: } 20 \\
\text { influenza: } 25 \\
\text { other: } 12\end{array}$ & $1987-96$ & & $\begin{array}{l}1.14 \\
(1.04-1.24)^{3}\end{array}$ \\
\hline $\begin{array}{l}\text { Larson et al. [56] } \\
\text { RUE: * } \\
\text { ROE: * }\end{array}$ & $\begin{array}{l}\text { western } \\
\text { Norway }\end{array}$ & hospital records searched for cases & NINCDS & 109 & all: 57 & $1957-82$ & all & $1.19^{3}$ \\
\hline $\begin{array}{l}\text { Sridharan et al. [34] } \\
\text { RUE: * } \\
\text { ROE: * }\end{array}$ & $\begin{array}{l}\text { Grampian, } \\
\text { Orkney and } \\
\text { Shetland, } \\
\text { Scotland }\end{array}$ & $\begin{array}{l}\text { retrospective search of hospital records } \\
\text { and morbidity records using ICD- } 8354 \text {, } \\
\text { ICD-9 } 357.0 \text { and } 357.9 \text { codes; diagnosis } \\
\text { confirmed by neurologist }\end{array}$ & NINCDS & 36 & influenza: 42 & $1980-88$ & all & $\begin{array}{l}1.1 \\
(0.8-1.4)^{1}\end{array}$ \\
\hline $\begin{array}{l}\text { Cuadrado et al. [35] } \\
\text { RUE: * } \\
\text { ROE: * }\end{array}$ & Spain & $\begin{array}{l}\text { retrospective review of hospital records, } \\
\text { laboratory records and discharge files }\end{array}$ & NINCDS & 337 & $\begin{array}{l}\text { URI: } 31 \\
\text { GI: } 12\end{array}$ & $1985-97$ & $>19$ & $0.86^{3}$ \\
\hline $\begin{array}{l}\text { Cuadrado et al. [36] } \\
\text { RUE: * } \\
\text { ROE: * }\end{array}$ & Spain & $\begin{array}{l}\text { prospective population-based study } \\
\text { with cases reported to central units; } \\
\text { records of cases checked by neurologist }\end{array}$ & NINCDS & 98 & $\begin{array}{l}\text { all: } 62 \\
\text { URI: } 43 \\
\text { GI: } 10 \\
\text { other: } 9\end{array}$ & 1998-99 & $>19$ & $1.26^{3}$ \\
\hline $\begin{array}{l}\text { Sedano et al. }[50] \\
\text { RUE: }{ }^{*} \\
\text { ROE: }{ }^{*}\end{array}$ & $\begin{array}{l}\text { Cantabria, } \\
\text { Spain }\end{array}$ & $\begin{array}{l}\text { hospital medical records searched for } \\
\text { ICD- } 8 \text { code } 354\end{array}$ & NINCDS & 69 & $\begin{array}{l}\text { all: } 36 \\
\text { URI: } 23 \\
\text { GI: } 9 \\
\text { other: } 4\end{array}$ & $1975-88$ & all & $\begin{array}{l}0.95 \\
(0.73-1.18)^{23}\end{array}$ \\
\hline $\begin{array}{l}\text { Jiang et al. [37] } \\
\text { RUE: }{ }^{*} \\
\text { ROE: }{ }^{*}\end{array}$ & Sweden & $\begin{array}{l}\text { cases discharged from a first hospital } \\
\text { stay }\end{array}$ & $\begin{array}{l}\text { ICD-9 code } \\
357 \mathrm{~A}\end{array}$ & 2,257 & not given & $1978-93$ & all & $1.77^{3,6}$ \\
\hline $\begin{array}{l}\text { Cheng et al. [38] } \\
\text { RUE: * } \\
\text { ROE: * }\end{array}$ & Sweden & $\begin{array}{l}\text { prospective data collection of newly } \\
\text { reported cases identified by neurolo- } \\
\text { gists; inpatient registries checked for } \\
\text { those with ICD-9 code } 357 \mathrm{~A}\end{array}$ & NINCDS & 73 & not given & 1996 & all & $\begin{array}{l}1.51 \\
(1.18-1.90)^{3}\end{array}$ \\
\hline $\begin{array}{l}\text { Jiang et al. [48] } \\
\text { RUE: * } \\
\text { ROE: * }\end{array}$ & $\begin{array}{l}\text { Stockholm } \\
\text { County, } \\
\text { Sweden }\end{array}$ & $\begin{array}{l}\text { medical records searched for } \\
\text { appropriate ICD codes }\end{array}$ & $\begin{array}{l}\text { codes ICD-8 } \\
354.01 \text { ICD-9 } \\
357 \mathrm{~A}\end{array}$ & 556 & not given & $1973-91$ & all & $\begin{array}{l}1.89 \\
(1.72-2.03)^{1,2}\end{array}$ \\
\hline $\begin{array}{c}\text { Jiang et al. [49] } \\
\text { RUE: * } \\
\text { ROE: * }\end{array}$ & $\begin{array}{l}\text { south-west } \\
\text { Stockholm, } \\
\text { Sweden }\end{array}$ & as above & as above & 84 & not given & $1973-91$ & all & $\begin{array}{l}1.56 \\
(1.241 .93)^{3}\end{array}$ \\
\hline
\end{tabular}


Table 1 (continued)

\begin{tabular}{|c|c|c|c|c|c|c|c|c|}
\hline Study & Location & Study method & $\begin{array}{l}\text { Diagnostic } \\
\text { criteria }\end{array}$ & $\begin{array}{l}\text { Cases } \\
\mathrm{n}\end{array}$ & $\begin{array}{l}\text { Antecedent } \\
\text { infection, } \%\end{array}$ & Period & Age & $\begin{array}{l}\text { Rate } \\
100,000 / \text { year }\end{array}$ \\
\hline \multicolumn{9}{|l|}{ The Middle East } \\
\hline $\begin{array}{l}\text { Arami et al. }[20] \\
\text { RUE: }{ }^{*} \\
\text { ROE: }{ }^{*}\end{array}$ & $\begin{array}{l}\text { Eastern Azer- } \\
\text { baijan, Iran }\end{array}$ & $\begin{array}{l}\text { prospective survey of all new cases of } \\
\text { GBS admitted to } 3 \text { referral centres }\end{array}$ & NINCDS & 76 & $\begin{array}{l}\text { all: } 65.8 \\
\text { URI: } 50 \\
\text { GI: } 16\end{array}$ & 2003 & all & $\begin{array}{l}2.11 \\
(1.64-2.59)^{2,3}\end{array}$ \\
\hline $\begin{array}{l}\text { Radhakrishnan et al. [19] } \\
\text { RUE: }{ }^{*} \\
\text { ROE: }{ }^{*}\end{array}$ & $\begin{array}{l}\text { Benghazi, } \\
\text { Libya }\end{array}$ & $\begin{array}{l}\text { screening through clinics, hospitals and } \\
\text { other centres identified cases }\end{array}$ & not given & 27 & all: 26 & $1983-85$ & all & $1.73^{3}$ \\
\hline \multicolumn{9}{|l|}{ North America and Canada } \\
\hline $\begin{array}{l}\text { Hauck et al. [39] } \\
\text { RUE: }{ }^{* *} \\
\text { ROE: }{ }^{* *}\end{array}$ & $\begin{array}{l}\text { Alberta, } \\
\text { Canada }\end{array}$ & $\begin{array}{l}\text { administrative sources maintained by } \\
\text { the Ministry of Health and Wellness of } \\
\text { the Government of Alberta }\end{array}$ & $\begin{array}{l}\text { codes used to } \\
\text { find cases }\end{array}$ & 496 & not given & $1994-04$ & all & 1.14 \\
\hline $\begin{array}{l}\text { McLean et al. [40] } \\
\text { RUE: * } \\
\text { ROE: * }\end{array}$ & $\begin{array}{l}\text { Quebec and } \\
\text { Ontario, } \\
\text { Canada }\end{array}$ & $\begin{array}{l}\text { hospital discharge databases searched; } \\
\text { sample of cases checked by } 2 \text { neurolo- } \\
\text { gists }\end{array}$ & $\begin{array}{l}\text { ICD }-9 \text { codes } \\
357.0,357.8 \\
357.9 \text { and } 375.0\end{array}$ & 2,333 & not given & $\begin{array}{l}1983-89 \\
\text { Ontario } \\
\text { Quebec }\end{array}$ & all & $\begin{array}{l}1.51 \\
1.78\end{array}$ \\
\hline $\begin{array}{l}\text { Alshekhlee et al. [45] } \\
\text { RUE: }{ }^{* *} \\
\text { ROE: }{ }^{* *}\end{array}$ & USA & $\begin{array}{l}\text { identified cohort from the Nationwide } \\
\text { Inpatient Sample which records data } \\
\text { from approx. } 20 \% \text { of community hospi- } \\
\text { tals }\end{array}$ & $\begin{array}{l}\text { included: ICD-9 } \\
\text { codes for GBS } \\
(357.0) \text {; excluded: } \\
357.81,357.82, \\
359.81,357.4, \\
045,045.1,358.0 \text {, } \\
358.01,358.1, \\
358.8,323,303.0 \text {, } \\
960,979\end{array}$ & 4,956 & not given & $2000-04$ & $>17$ & $1.72^{2}$ \\
\hline $\begin{array}{l}\text { Kaplan et al. [65] } \\
\text { RUE: * } \\
\text { ROE: * }\end{array}$ & $\begin{array}{l}\text { Colorado, } \\
\text { USA }\end{array}$ & retrospective review of medical records & $\begin{array}{l}\text { diagnosed by a } \\
\text { neurologist; } \\
\text { records with } \\
\text { ICD-9 } 357.0 \\
\text { reviewed }\end{array}$ & 48 & $\begin{array}{l}\text { all (Larimer } \\
\text { County): } 86 \\
\text { URI } 53 \\
\text { GI } 23 \\
\text { other } 10\end{array}$ & $\begin{array}{l}1975-83 \\
\text { Larimer } \\
\text { County } \\
\text { Weld } \\
\text { County }\end{array}$ & all & $\begin{array}{l}2.20 \\
(1.41-3.02)^{1,2} \\
1.80 \\
(0.97-2.55)^{1,2}\end{array}$ \\
\hline $\begin{array}{l}\text { Church Potter et al. [41] } \\
\text { RUE: }{ }^{*} \\
\text { ROE: }{ }^{* *}\end{array}$ & $\begin{array}{l}\text { Michigan, } \\
\text { USA }\end{array}$ & $\begin{array}{l}\text { data on GBS cases collected retrospec- } \\
\text { tively from the community health } \\
\text { reportable disease database and GBS } \\
\text { registry }\end{array}$ & $\begin{array}{l}\text { diagnostic } \\
\text { criteria not } \\
\text { given }\end{array}$ & 471 & not given & $1992-99$ & & 0.63 \\
\hline $\begin{array}{l}\text { Beghi et al. [66] } \\
\text { RUE: * } \\
\text { ROE: * }\end{array}$ & $\begin{array}{l}\text { Olmsted } \\
\text { county, } \\
\text { USA }\end{array}$ & $\begin{array}{l}\text { retrospective review of medical records } \\
\text { of the Mayo Clinic record-linkage sys- } \\
\text { tem; records evaluated and diagnosis } \\
\text { confirmed by neurologists }\end{array}$ & $\begin{array}{l}\text { codes identified } \\
\text { included GBS, } \\
\text { polyradiculitis, } \\
\text { peripheral neuri- } \\
\text { tis or neuronitis }\end{array}$ & 48 & all 65 & $1935-80$ & all & $1.77^{3}$ \\
\hline $\begin{array}{l}\text { Hoppock et al. [42] } \\
\text { RUE: }{ }^{*} \\
\text { ROE: }{ }^{*}\end{array}$ & $\begin{array}{l}\text { Sedgwick } \\
\text { County, } \\
\text { Kansas, } \\
\text { USA }\end{array}$ & hospital records were reviewed & $\begin{array}{l}\text { ICD code } 357.0 \\
\text { and clinical fea- } \\
\text { tures }\end{array}$ & 43 & $\begin{array}{l}\text { all: } 73 \\
\text { URI: } 47 \\
\text { GI: } 21 \\
\text { URI and GI: } 5\end{array}$ & $1984-88$ & all & 2.2 \\
\hline $\begin{array}{l}\text { Koobatian et al. [43] } \\
\text { RUE: * } \\
\text { ROE: * }\end{array}$ & $\begin{array}{l}\text { Vermont, } \\
\text { USA }\end{array}$ & $\begin{array}{l}\text { computer discharge abstracts from all } \\
\text { hospitals searched for GBS ICD-9 code } \\
357.0\end{array}$ & NINCDS & 51 & all: 57 & $1980-85$ & & 1.6 \\
\hline $\begin{array}{l}\text { Riggs et al. [51] } \\
\text { RUE: ** } \\
\text { ROE: * }\end{array}$ & $\begin{array}{l}\text { West Virginia, } \\
\text { USA }\end{array}$ & $\begin{array}{l}\text { medical records of patients admitted } \\
\text { with acute neuropathies reviewed }\end{array}$ & not given & 92 & not given & $1967-87$ & all & $1.7^{3}$ \\
\hline \multicolumn{9}{|l|}{ South America } \\
\hline $\begin{array}{l}\text { Rocha et al. [15] } \\
\text { RUE: *** } \\
\text { ROE: }{ }^{*}\end{array}$ & $\begin{array}{l}\text { Sao Paulo, } \\
\text { Brazil }\end{array}$ & $\begin{array}{l}\text { medical record review (1995-1999) } \\
\text { and clinical registration of cases } \\
\text { (2000-2002); all cases examined by } \\
\text { neurological staff }\end{array}$ & $\begin{array}{l}\text { Asbury and } \\
\text { Cornblath }\end{array}$ & 95 & $\begin{array}{l}\text { all: } 58 \\
\text { URI: } 46 \\
\text { GI: } 12\end{array}$ & 1995-2002 & all & $\begin{array}{l}0.40 \\
(0.32-0.48)^{1,2}\end{array}$ \\
\hline
\end{tabular}

Figures in parentheses are $95 \%$ CI.

RUE $=$ Risk of underestimation; ROE $=$ risk of overestimation; ${ }^{*}=$ low; ${ }^{*}=$ medium; ${ }^{* *}=$ high; MF $=$ Miller-Fisher; URI $=$ upper respiratory tract infection; $\mathrm{GI}=$ gastrointestinal infection.

${ }^{1}$ Rate checked and confirmed. ${ }^{2} \mathrm{CI}$ added. ${ }^{3}$ Standardised rate. ${ }^{4}$ Date used in incidence calculations is date of diagnosis. ${ }^{5}$ Rate calculated from number of cases and population data provided in the paper. ${ }^{6}$ Date used in incidence calculations is date of first admission to hospital. 


\section{Clinical Variants of GBS}

Very few studies included in this review gave information about incidence of the clinical variants of GBS - of those studies that did, the main variant of GBS, MillerFisher syndrome, was much less common than GBS. If cases of these diseases were included in any studies of GBS incidence, then the overall incidence rate would only be overestimated by a small amount.

\section{Antecedent Infection}

A number of the studies included in this review gave details of numbers of cases reporting an antecedent infection, usually less than 4 weeks, before onset of GBS. These data have been recorded in table 1 along with a breakdown of the type of infection, where given. In most studies reporting this information, $40-70 \%$ of cases recorded an infection before onset with $22-53 \%$ having an upper respiratory tract infection and $6-26 \%$ a gastrointestinal infection. Children's rates were higher, with $67-85 \%$ of all cases reporting an infection: $50-70 \%$ were respiratory infections and $7-14 \%$ gastrointestinal infections.

\section{Seasonal Variation}

The issue of seasonal variation in incidence was raised in some studies although none reported significant differences in levels of onset of GBS between seasons [16, 28, $29,50,52-54]$. Some found more cases in colder months $[9,19,20,46,55,56]$ although a cluster of cases was reported in spring and summer in Brazil [15], during the winter and June in the Netherlands [33] and during autumn in Sweden [37].

\section{Study Method}

Study design is an important consideration when comparing incidence rates between different studies. In this review, the studies included were of 3 types: prospective [11-13, 20, 24, 27, 29-32, 36, 38, 47, 53, 54, 57-59], retrospective medical record review $[9,14-19,21,25,26,33-$ $35,41,46,50-52,55,56,60-66]$ and retrospective database studies with $[8,10,40,67,68]$ or without $[22,23,37$, $39,42-45,48,49]$ the review of cases by a clinician. At first glance, it would appear that study design did not have a sizeable effect as the incidence rates found were similar. However, when incidence rates were compared by type of study, some trends became apparent. For the prospective studies, the majority of incidence rates were between 1.11/100,000/year and 1.66/100,000/year; for the retrospective record review studies and the database studies with record review, most were between 0.83 / $100,000 /$ year and $1.77 / 100,000$ /year and for the database studies where a review of cases was not performed, 1.14/ $100,000 /$ year to $2.2 / 100,000$ /year. Prospective studies are usually the most reliable; using this as the standard suggests that some of the database studies overestimated incidence rate and some of the retrospective record review studies underestimated rates.

Most patients diagnosed with GBS will be hospitalised due to the nature of the disease $[8,26,32,33,35-37,64]$ : 3 studies reported small numbers of outpatient or community patients identified in their studies compared with numbers of hospitalised GBS cases [12, 32, 38]. Ten studies noted the inclusion of mild cases in their studies [16, $24,29,33,38,45,46,50,57,59]$ and the design of other studies implied the inclusion of mild cases: 2 studies using general practitioner records [22, 23], a surveillance study [54], a door-to-door survey [58] and the record linkage system used by the Mayo Clinic [66]. Of those studies that used mainly hospital records, 2 noted that all patients were given intravenous immunoglobulin or plasma exchange therapy [20,67], and this implies that only serious cases were included, whereas others reported proportions of patients receiving these therapies of between 24 and $49 \%$ for intravenous immunoglobulin and $34-$ $51 \%$ receiving plasma exchange $[26,27,30,47,61]$. Ten studies reported missing mild cases $[12,32,36,64,69,70]$ or reported finding it difficult to detect mild cases if they had not been admitted to hospital $[17,25,37,62]$.

\section{Discussion}

Most of the incidence rates of GBS reported were between 1.1/100,000/year and 1.8/100,000/year with lower rates reported in children ( $<16$ years) of $0.4 / 100,000 /$ year to $1.4 / 100,000 /$ year. Most of the studies included were from Europe and North America where the rates found were similar.

A number of studies have commented on a bimodal pattern of incidence by age, with peaks occurring in young adults and the elderly [3, 24, 71]. In this review, only 1 study [19] (out of 24) found a peak in incidence in young adults, although the rates were not adjusted and a high proportion of young adults in this area could have biased the incidence rates found.

The majority of studies used the recognised NINCDS criteria or a comparable set of diagnostic criteria, and this allowed comparisons to be made between studies. One important difference between studies that should be highlighted is the study method used. In this review, the overall incidence rates found by the prospective studies 


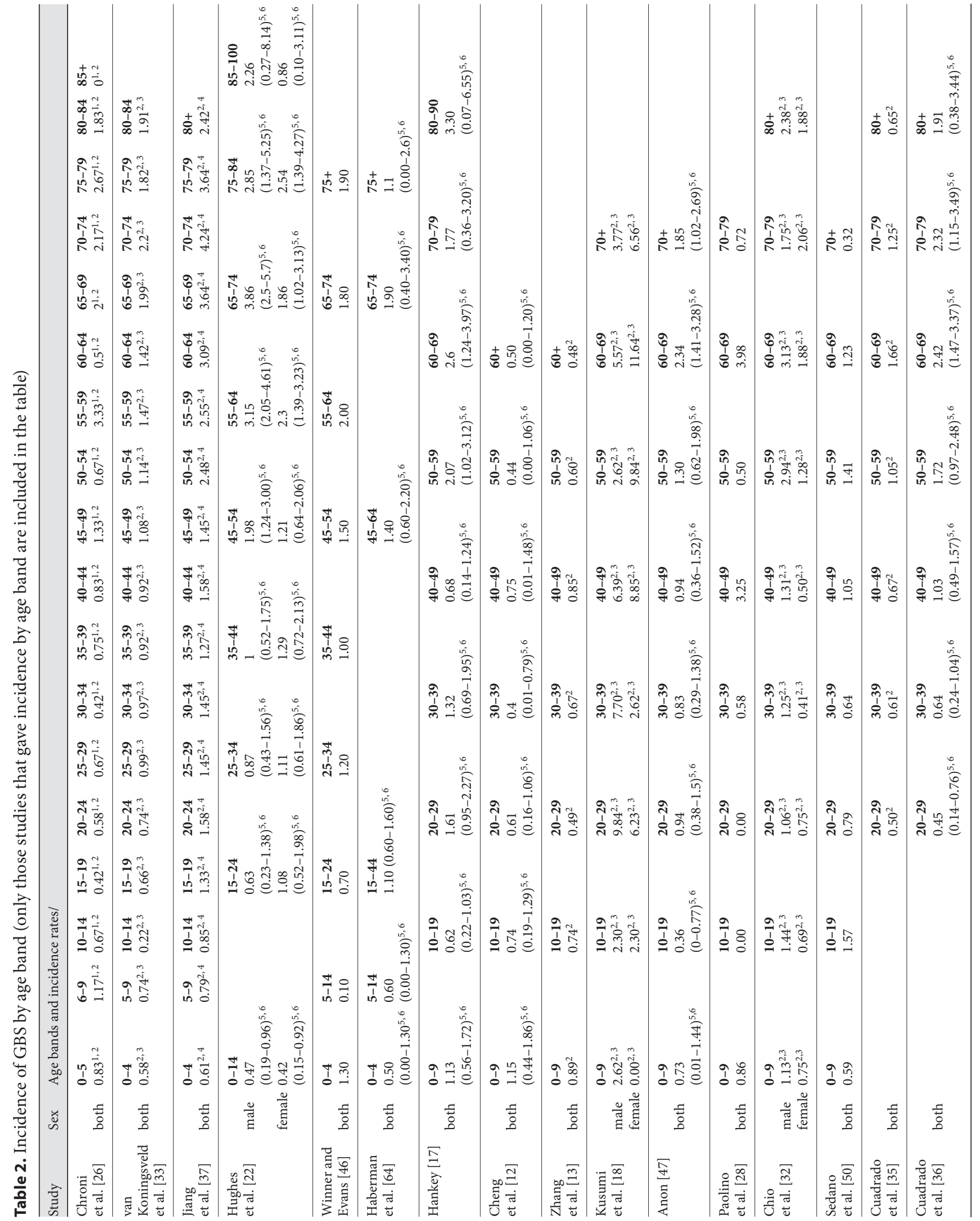




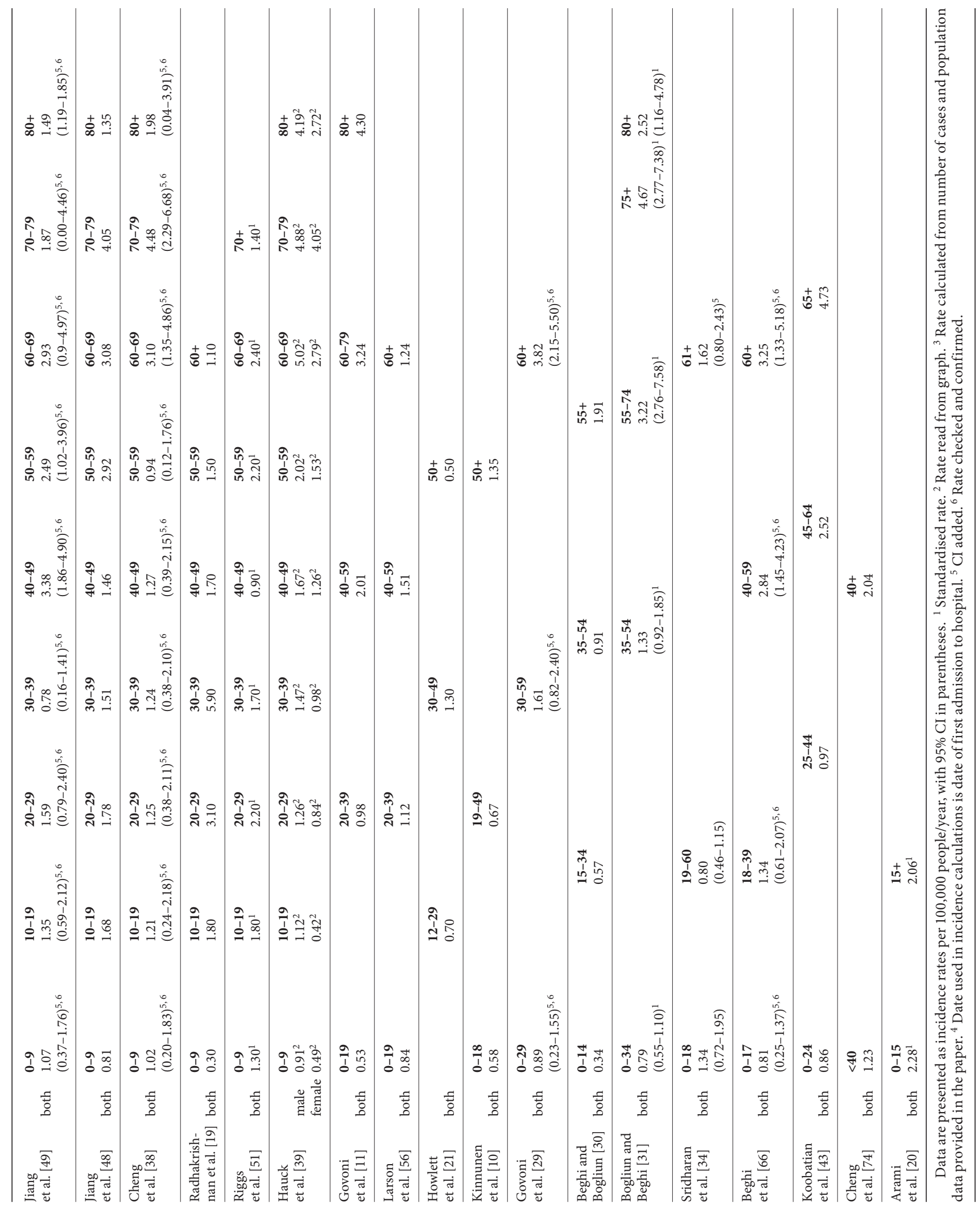


and the database studies that did not review cases were higher than those found by the retrospective studies that reviewed medical records. Prospective studies of incidence are thought to be the most accurate provided the ascertainment of cases and determination of denominator are reliable. This is thought to be true of the prospective studies included in this review, so the incidence rates from these are likely to be the best approximation available for the true incidence of GBS. The range of incidence rates found by the retrospective studies where medical records were reviewed produced slightly lower incidence rates, which indicates that some cases may have been missed by these studies. Conversely, it is possible that the studies using databases where medical records were not reviewed overestimated the incidence rates. Bogliun et al. [72] compared case ascertainment between a hospital discharge database and a registry, and found that over half of the 'cases' identified in the discharge database had either not had their diagnosis confirmed or had been double-counted through re-admission to hospital. The possibility of inaccurate coding could also contribute to overestimation of cases in this type of study. The database studies did not provide any indication of the criteria used when the original diagnosis was made, which is more likely to be a feature of this particular study method than a lack of regard for set diagnostic guidelines.

The identity of GBS as a single homogenous clinical entity is evolving and reference to GBS as a term covering the disease subtypes AIDP, AMAN, AMSAN and MillerFisher syndrome is becoming more common [71, 73]. GBS also has links with chronic diseases, including chronic inflammatory demyelinating neuropathy and subacute demyelinating polyneuropathy [71]. Possible implications for interpretation of incidence rates in epidemiological studies of GBS are that most of the studies included in this review used the NINCDS criteria, which do not include the symptoms of Miller-Fisher syndrome but do include AIDP, AMAN and AMSAN. Therefore, if Miller-Fisher syndrome is to be included in the definition, then some of the rates reported here derived from studies using NINCDS criteria will be slight underestimates of the true incidence. Geographical patterns of incidence of AIDP, AMAN and AMSAN have been reported $[5,73]$. Very few studies in this review included information on the incidence of variants of GBS, and those that did concentrated on whether cases of Miller-Fisher syndrome were included; it was not possible to determine whether there was a geographical difference between incidence of AIDP, AMAN and AMSAN. A link between the incidence of clinical variants and preceding infection was made by Govoni et al. [29] who compared GBS incidence with clinical variants of GBS including MillerFisher syndrome, polyneuritis cranialis, sensory form, acute pandysautonomia and chronic inflammatory demyelinating polyneuropathy. They found that a higher number of cases with preceding infection amongst individuals with clinical variants than amongst those with GBS ( $88 \%$ compared with $54 \%$ in their study), and that the disease was milder at nadir for clinical variants of GBS. Some investigators recommend that the NINCDS criteria are widened to include clinical variants and this might result in more cases being identified $[29,66]$.

The association with antecedent viral and upper respiratory infections before onset of GBS has been known for over 100 years, although the suggested link between Campylobacter jejuni and GBS is much more recent [73]. Potential links have been reported between Campylobacter jejuni and axonal forms of GBS [73]. It is acknowledged that the reporting of antecedent infections is likely to be more accurate in prospective than in retrospective studies; for example less than $40 \%$ of cases of GBS were reported to be preceded by an infection in 3 retrospective studies in Italy $[28,31,55]$ whereas a prospective study from Italy reported a rate of preceding infection of $70 \%$ $[27,47]$. It is therefore reasonable to assume that most cases of GBS are triggered by antecedent infection, although no indications were given as to the likely cause of the majority of the remaining cases. Hughes and Rees [4] noted that the lack of obvious seasonal changes in incidence may be because the infections found most frequently to trigger this disease, respiratory and enteric infections, have opposite seasonality of occurrence. Two studies investigated potential seasonal variations in cases for those who reported an antecedent infection before onset of GBS: Larson et al. [56] found seasonal variation to be more pronounced in this group, but Paolino et al. [28] did not find a significant association with onset in cold or warm months.

\section{Conclusions}

Overall the best estimate of the incidence of GBS is between $1.1 / 100,000$ /year and 1.8/100,000/year with lower rates reported in children ( $<16$ years) of around $0.6 / 100,000 / y e a r$. The review reported mostly on studies from Europe and North America. Few rates were presented from other parts of the world, particularly Asia and Africa, and this makes it difficult to comment on possible geographical variations. Incidence increased 
with age from 50 years; we were unable to confirm the suggestion that bimodality exists in the incidence of GBS. Antecedent infections, mainly upper respiratory and gastrointestinal infections, preceded up to $70 \%$ of cases reported.

The widespread use of the NINCDS criteria provides consistency across studies. Differences in incidence rates between studies with different methods were found: pro- spective studies were thought to be the most reliable; however, large differences in incidence rates between the various types of studies were not found.

\section{Acknowledgements}

This work was supported by a grant from GSK Biologicals.

\section{Appendix}

Data abstraction forms:

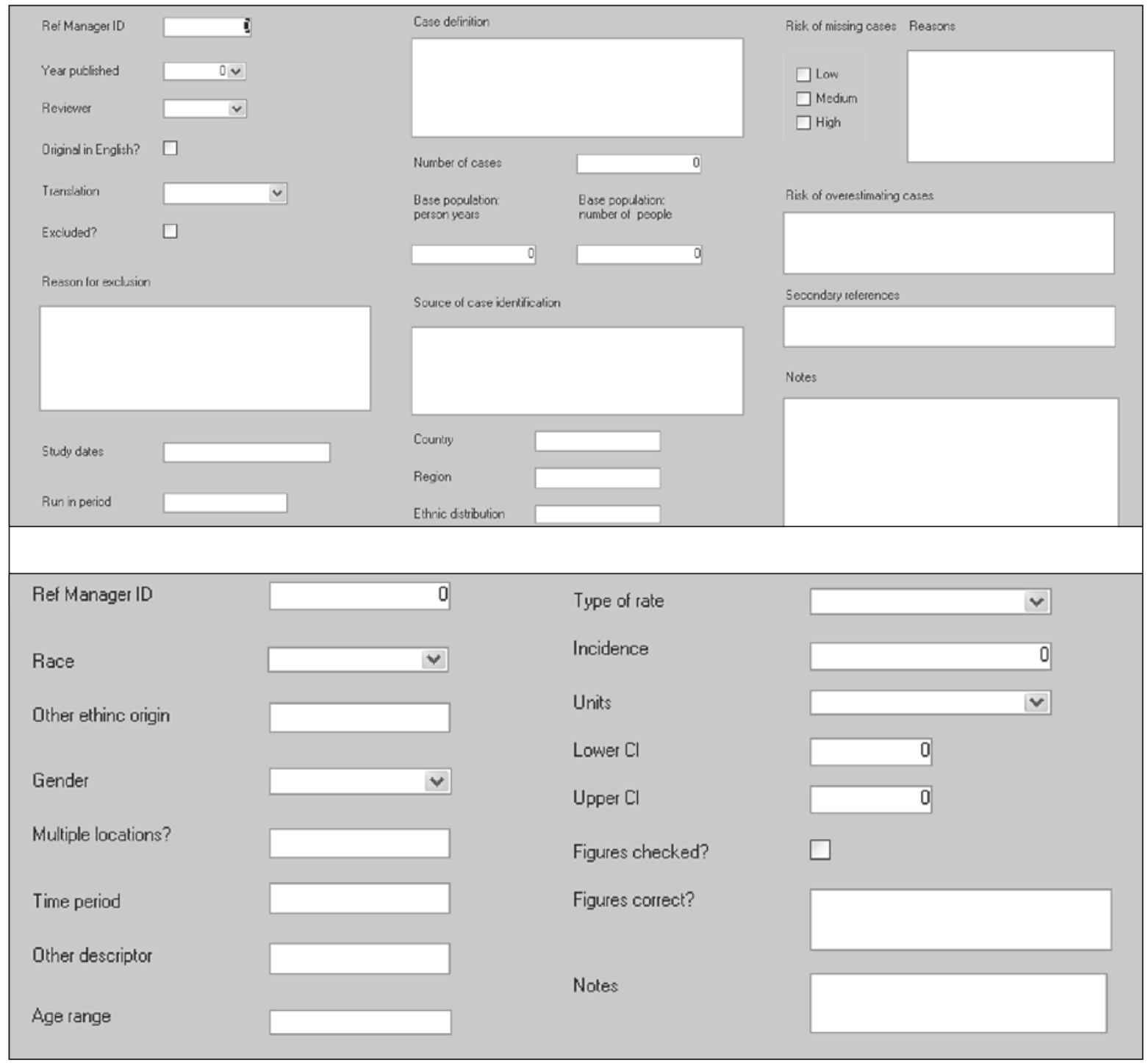




\section{References}

1 Hughes RA, Rees JH: Clinical and epidemiologic features of Guillain-Barre syndrome. J Infect Dis 1997;176:92-98.

-2 Asbury AK, Arnason BG, Karp HR, McFarlin DE: Criteria for diagnosis of GuillainBarre syndrome. Ann Neurol 1978;3:565566.

$\checkmark 3$ Govoni V, Granieri E: Epidemiology of the Guillain-Barre syndrome. Curr Opin Neurol 2001; 14:605-613

$\checkmark 4$ Hughes RAC, Rees JH: Clinical and epidemiologic features of Guillain-Barre syndrome. J Infect Dis 1997;176(suppl 2):S92S98.

5 Hughes RA, Cornblath DR: Guillain-Barre syndrome. Lancet 2005;366:1653-1666.

6 Tsai CP, Wang KC, Liu CY, Sheng WY, Lee TC: Pharmacoeconomics of therapy for Guillain-Barre syndrome: plasma exchange and intravenous immunoglobulin. J Clin Neurosci 2007; 14:625-629.

7 Sladky JT: Guillain-Barre syndrome in children. J Child Neurol 2004;19:191-200.

$\checkmark 8$ Rantala H, Uhari M, Niemela M: Occurrence, clinical manifestation and prognosis of Guillain-Barre syndrome. Arch Dis Child 1991;66:706-708.

$\checkmark 9$ van Koningsveld R, Rico R, Gerstenbluth I, Schmitz PI, Ang CW, Merkies IS, et al: Gastroenteritis-associated Guillain-Barre syndrome on the Caribbean island Curacao. Neurology 2001;56:1467-1472.

-10 Kinnunen E, Junttila O, Haukka J, Hovi T: Nationwide oral poliovirus vaccination campaign and the incidence of GuillainBarre Syndrome. Am J Epidemiol 1998;147: 69-73.

11 Govoni V, Granieri E, Manconi M, Capone J, Casetta I: Is there a decrease in GuillainBarre syndrome incidence after bovine ganglioside withdrawal in Italy? A populationbased study in the Local Health District of Ferrara, Italy. J Neurol Sci 2003;216:99-103.

12 Cheng Q, Wang DS, Jiang GX, Han H, Zhang Y, Wang WZ, et al: Distinct pattern of agespecific incidence of Guillain-Barre syndrome in Harbin, China. J Neurol 2002;249: 25-32.

13 Zhang Y, Wang DS, Han H, Li F, Sheng L, Link H: Epidemiological survey of the incidence of Guillain-Barre syndrome in Harbin from 1997 to 1999. Chin J Clin Rehab 2004; 7812-7815.

$\checkmark 14$ Hui AC, Chow KM, Tang AS, Fu M, Kay R, Wong KS: Electrophysiological, clinical and epidemiological study of Guillain-Barre Syndrome in Hong Kong Chinese. J Clin Neurosci 2005;12:134-136.

-15 Rocha MS, Brucki SM, Carvalho AA, Lima UW: Epidemiologic features of GuillainBarre syndrome in Sao Paulo, Brazil. Arq Neuropsiquiatr 2004;62:33-37.
16 Storey E, Cook M, Peppard R, Newton-John $\mathrm{H}$, Byrne E: Guillain-Barre syndrome and related conditions in Victorian teaching hospitals 1980-1984. Aust NZ J Med 1989;19: 687-693.

17 Hankey JG: Guillain-Barre syndrome in Western Australia, 1980-1985. Med J Aust 1987;146:130-133.

18 Kusumi M, Nakashima K, Nakayama H, Takahashi K: Epidemiology of inflammato ry neurological and inflammatory neuromuscular diseases in Tottori Prefecture, Japan. Psychiatry Clin Neurosci 1995;49: 169-174

19 Radhakrishnan K, El-Mangoush MA, Rerryo SE: Descriptive epidemiology of selected neuromuscular disorders in Benghazi, Libya. Acta Neurol Scand 1987;75:95-100.

20 Arami MA, Yazdchi M, Khandaghi R: Epidemiology and characteristics of GuillainBarre syndrome in the northwest of Iran. Ann Saudi Med 2006;26:22-27.

21 Howlett WP, Vedeler CA, Nyland H, Aarli JA: Guillain-Barre syndrome in northern Tanzania: a comparison of epidemiologic and clinical findings with western Norway. Acta Neurol Scand 1996;93:44-49.

22 Hughes RA, Charlton J, Latinovic R, Gulliford MC: No association between immunization and Guillain-Barre syndrome in the United Kingdom, 1992 to 2000. Arch Intern Med 2006;166:1301-1304

23 MacDonald BK, Cockerell OC, Sander JWAS, Shorvon SD: The incidence and lifetime prevalence of neurological disorders in a prospective community-based study in the UK. Brain 2000;123:665-676.

24 ReesJH, Thompson RD, Smeeton NC, Hughes RAC: Epidemiological study of GuillainBarre syndrome in south east England. J Neurol Neurosurg Psychiatry 1998;64:74-77.

25 Markoula S, Giannopoulos S, Sarmas I, Tzavidi S, Kyritsis AP, Lagos G: Guillain-Barre syndrome in northwest Greece. Acta Neurol Scand 2007;115:167-173.

26 Chroni E, Papapetropoulos S, Gioldasis G, Ellul J, Diamadopoulos N, Papapetropoulos T: Guillain-Barre syndrome in Greece: seasonality and other clinico-epidemiological features. Eur J Neurol 2004;11:383-388.

27 Guillain-Barre syndrome variants in Emilia-Romagna, Italy, 1992-3: incidence, clinical features, and prognosis. Emilia-Romagna Study Group on Clinical and Epidemiological Problems in Neurology. J Neurol Neurosurg Psychiatry 1998;65:218-224.

28 Paolino Z, Govoni V, Tola MR, Casetta I, Granieri E: Incidence of the Guillain-Barre syndrome in Ferrara, Northern Italy, 19811987. Neuroepidemiology 1991;10:105-111.

29 Govoni V, Granieri E, Tola MR, Casetta I, Ruppi P, Vaghi L: The frequency of clinical variants of Guillain-Barre syndrome in Ferrara, Italy. J Neurol 1999;246:1010-1014.
30 Beghi E, Bogliun G: The Guillain-Barre syndrome (GBS): implementation of a register of the disease on a nationwide basis. Ital J Neurol Sci 1996;17:355-361.

-31 Bogliun G, Beghi E, Italian GBS Registry Study Group: Incidence and clinical features of acute inflammatory polyradiculoneuropathy in Lombardy, Italy, 1996. Acta Neurol Scand 2004;110:100-106.

32 Chio A, Cocito D, Leone M, Giordana MT, Mora G, Mutani R, et al: Guillain-Barre syndrome: a prospective, population-based incidence and outcome survey. Neurology 2003;60:1146-1150.

-33 van Koningsveld R, van Doorn PA, Schmitz PI, Ang CW, van der Meche FG: Mild forms of Guillain-Barre syndrome in an epidemiologic survey in The Netherlands. Neurology 2000;54:620-625.

34 Sridharan GV, Tallis RC, Gautam PC: Guillain-Barre syndrome in the elderly: a retrospective comparative study. Gerontology 1993;39:170-175.

35 Cuadrado JI, Pedro-Cuesta J, Ara JR, Cemillan CA, Diaz M, Duarte J, et al: GuillainBarre syndrome in Spain, 1985-1997: epidemiological and public health views. Eur Neurol 2001;46:83-91.

-36 Cuadrado JI, Pedro-Cuesta J, Ara JR, Cemillan CA, Diaz M, Duarte J, et al: Public health surveillance and incidence of adulthood Guillain-Barre syndrome in Spain, 1998-1999: the view from a sentinel network of neurologists. Neurol Sci 2004;25:57-65.

37 Jiang GX, Cheng Q, Link H, de Pedro-Cuesta J: Epidemiological features of GuillainBarre syndrome in Sweden 1978-1993. J Neurol Neurosurg Psychiatry 1997;62:447453.

38 Cheng Q, Jiang GX, Fredrikson S, Link H, Pedro-Cuesta J: Incidence of Guillain-Barre syndrome in Sweden 1996. Eur J Neurol 2000;7:11-16.

39 Hauck LJ, White C, Feasby TE, Zochodne DW, Svenson LW, Hill MD: Incidence of Guillain-Barre syndrome in Alberta, Canada: an administrative data study. J Neurol Neurosurg Psychiatry 2008;79:318-320.

40 McLean M, Duclos P, Jacob P, Humphreys P: Incidence of Guillain-Barre syndrome in Ontario and Quebec, 1983-1989, using hospital services databases. Epidemiology 1994; 5:443-448.

41 Church Potter R, Kaneene JB: A descriptive study of Guillain-Barre syndrome in high and low Campylobacter jejuni incidence regions of Michigan: 1992-1999. Neuroepidemiology 2003;22:245-248.

42 Hoppock KC, Greer GG, Walling AD: The incidence of Guillain-Barre syndrome in a metropolitan county, 1984-1988. Kans Med 1994;95:45-47. 
43 Koobatian TJ, Birkhead GS, Schramm MM, Vogt RL: The use of hospital discharge data for public health surveillance of GuillainBarre syndrome. Ann Neurol 1991;30:618621.

44 Lehmann HC, Köhne A, Meyer zu Hörste G, Kieseier BC: Incidence of Guillain-Barre syndrome in Germany. J Peripher Nerv Syst 2007;12:285.

-45 Alshekhlee A, Hussain Z, Sultan B, Katirji B: Guillain-Barre syndrome: incidence and mortality rates in US hospitals. Neurology 2008;70:1608-1613.

-46 Winner SJ, Evans JG: Age specific incidence of Guillain-Barre syndrome in Oxfordshire. Q J Med 1990;77:1297-1304.

47 A prospective study on the incidence and prognosis of Guillain-Barre syndrome in Emilia-Romagna region, Italy (1992-1993). Emilia-Romagna Study Group on Clinical and Epidemiological Problems in Neurology. Neurology 1997;48:214-221.

-48 Jiang GX, Cheng Q, Ehrnst A, Link H, de Pedro-Cuesta J: Guillain-Barre syndrome in Stockholm county, 1973-1991. Eur J Epidemiol 1997; 13:25-32.

-49 Jiang GX, de Pedro-Cuesta J, Fredrikson S: Guillain-Barre syndrome in south-west Stockholm, 1973-1991. 1. Quality of registered hospital diagnoses and incidence. Acta Neurol Scand 1995;91:109-117.

-50 Sedano MJ, Calleja J, Canga E, Berciano J: Guillain-Barre syndrome in Cantabria, Spain: an epidemiological and clinical study. Acta Neurol Scand 1994;89:287-292.

51 Riggs JE, Cutmann L, Whited JD: GuillainBarre syndrome: another immune-mediated disease with a predilection for young women? W V Med J 1989;85:382-383.

52 D’Ambrosio G, De Angelis G, Vizoli R: Epidemiology of Guillain-Barre syndrome in Campania (South Italy). Acta Neurol 1983; 23:245-252.
53 Barzegar M, Dastgiri S, Karegarmaher MH, Varshochiani A: Epidemiology of childhood Guillan-Barre syndrome in the north west of Iran. BMC Neurology 2007;7:22.

54 Dias-Tosta E, Kuckelhaus CS: Guillain Barre syndrome in a population less than 15 years old in Brazil. Arq Neuropsiquiatr 2002;60: 367-373.

55 Congia S, Melis M, Carboni MA: Epidemiologic and clinical features of the GuillainBarre syndrome in Sardinia in the 1961-1980 period. Acta Neurol 1989;11:15-20.

56 Larson JP, Kvale G, Nyland H: Epidemiology of the Guillain-Barre syndrome in the county of Hordaland, Western Norway. Acta Neurol Scand 1985;71:43-47.

57 Molinero MR, Varon D, Holden KR, Sladky JT, Molina IB, Cleaves F: Epidemiology of childhood Guillain-Barre syndrome as a cause of acute flaccid paralysis in Honduras: 1989-1999. J Child Neurol 2003;18:741-747.

58 Olive JM, Castillo C, Garcia Castro R, de Quadros CA: Epidemiologic study of Guillain-Barre syndrome in children $<15$ years of age in Latin America. J Infect Dis 1997; 175(suppl):S160-S164.

59 Hart DE, Rojas LA, Rosario JA, Recalde H, Roman GC: Childhood Guillain-Barre syndrome in Paraguay, 1990 to 1991. Ann Neurol 1994;36:859-863.

60 Hung KL, Wang HS, Liou WY, Mak SC, Chi CS, Shen EY, et al: Guillain-Barre syndrome in children: a cooperative study in Taiwan. Brain Dev 1994;16:204-208.

61 Artan R: Childhood Guillain Barre syndrome in Antalya, Turkey. Int Med J 1997;4: 215-217.

62 Bak P: Guillain-Barre syndrome in a Danish county. Neurology 1985;35:207-211.

63 Halls J, Bredkjaer C, Friis ML: GuillainBarre syndrome: diagnostic criteria, epidemiology, clinical course and prognosis. Acta Neurol Scand 1988;78:118-122.

-64 Haberman S, Benjamin B, Rose FC, Capildeo $\mathrm{R}$ : North west Thames registry of neurological disease. J Royal Soc Med 1982;75:443449.
65 Kaplan JE, Poduska PJ, McIntosh GC, Hopkins RS, Ferguson SW, Schonberger LB: Guillain-Barre syndrome in Larimer County, Colorado: a high incidence area. Neurology 1985;35:581-584.

66 Beghi E, Kurland LT, Mulder DW, Wiederholt WC: Guillain-Barre syndrome: clinicoepidemiologic features and effect of influenza vaccine. Arch Neurol 1985;42:1053-1057.

67 Ismail EA, Shabani IS, Badawi M, Sanaa H, Madi S, Al Tawari A, et al: An epidemiologic, clinical, and therapeutic study of childhood Guillain-Barre syndrome in Kuwait: is it related to the oral polio vaccine? J Child Neurol 1998;13:488-492.

68 Rantala H, Cherry JD, Shields WD, Uhari M: Epidemiology of Guillain-Barre syndrome in children: relationship of oral polio vaccine administration to occurrence. J Pediatr 1994; 124:220-223.

69 Rantala H, Uhari M, Niemela M: Occurrence, clinical manifestations, and prognosis of Guillain-Barre syndrome. Arch Dis Child 1991;66:706-708.

70 Farkkila M, Kinnunen E, Weckstrom P: Survey of Guillain-Barre syndrome in southern Finland. Neuroepidemiology 1991;10:236241.

71 Winer JB: Guillain Barre syndrome. Mol Pathol 2001;54:381-385.

72 Bogliun G, Beghi E, Guillain-Barre Syndrome Registry Study Group: Validity of hospital discharge diagnoses for public health surveillance of the Guillain-Barre syndrome. Neurol Sci 2002;23:113-117.

73 Nachamkin I, Allos BM, Ho T: Campylobacter species and Guillain-Barre syndrome. Clin Microbiol Rev 1998;11:555-567.

74 Cheng Q, Jiang GX, Fredrikson S, Link H, Pedro-Cuesta J: Epidemiological surveillance of Guillain-Barre syndrome in Sweden, 1996-1997: network members of the Swedish GBS Epidemiology Study Group. Acta Neurol Scand 2000;101:104-110. 